\title{
ARTICLE
}

\section{Options for Controlling Localized Skin Dose}

\author{
Sang-Hyun PARK ${ }^{1 *}$, Jaiki LEE ${ }^{2}$ \\ ${ }^{l}$ National Research Foundation of Korea, 165, Gajeong-ro, Yuseong-gu, Daejeon 305-350, Korea \\ ${ }^{2}$ Hanyang University, Haengdang-dong, Sungdong-gu, Seoul 133-791, Korea
}

\begin{abstract}
In this study, issue on the skin dose averaging area was reviewed with the results of calculated dose distribution from various beta emitters. In case of dot-like contamination such as a hot particle, according to calculated results, over $99 \%$ of the dose was deposited within $1 \mathrm{~mm}$ radius. This result showed that the use of $10 \mathrm{~cm}^{2}$ as the dose averaging area, recommended by the National Council on Radiation Protection and Measurement (NCRP), compromises the concept of dosimetry by intentionally including unexposed tissue volume to reduce the dose. Beside the approach recommended by NCRP, two other options were suggested and discussed. One option addressing a graded enforcement of the skin dose limit, considering the low probability of hot particle exposure, was proposed to avoid compromising the dosimetry concept. Though this option can give flexibility in the regulation of skin exposure, this option compromises the unified meaning of the dose limits. The other option addressed increase of the skin equivalent dose limit specifically for prevention of deterministic effects while applying a smaller averaging area for controlling stochastic effects. The first two options involve decision of the national or regional regulatory authority and the third involves actions at the International Commission on Radiological Protection (ICRP) level.
\end{abstract}

KEYWORDS: skin dose, hot particle, dose averaging area, dosimetry, regulation

\section{Introduction}

Hot particles are small high-activity radioactive particles of nominal diameter ranging from $\sim 1 \mathrm{~mm}$ to several $\mu \mathrm{m} .{ }^{1)}$ The skin contamination by hot particles can be expected during decommissioning and routine operation in nuclear sites. $^{2)}$ As the most superficial organ of the body, skin can often receive the highest absorbed dose from an external exposure. It also has the highest radiation-induced cancer incidence risk factor of any organ. Therefore, exposures to hot particles, which give significantly high local dose on small area, are potential risks for both deterministic and stochastic effects.

However, proper evaluation of its hazards is difficult. This is partly due to the problems involved in estimating doses from the highly non-uniform dose distributions produced by such small radioactive particles and also to the problem of estimating the relative biological response compared with uniform exposures.

To cope with skin exposure from hot particles, local regulatory bodies and licensees have to establish reasonable and pragmatic procedures for monitoring hot particles, controlling of skin exposure and dose assessment based on reference value such as skin dose limits. The ICRP and NCRP in recent years have extensively reviewed the exposure of the skin on the basis of an extensive body of radiobiological, clinical and epidemiological data and set skin dose limits. ${ }^{3-4)}$ However, as Charles indicated, due to a divergence of philosophy regarding acceptability of deterministic effects, they used different dose averaging area to set skin dose limits. ${ }^{5}$ The disparity remained in the recommendations between the ICRP and the NCRP would

*Corresponding Author, E-mail: sanghyunpark01@gmail.com

(C) Atomic Energy Society of Japan perplex local regulatory bodies and licensees.

In this study, issue on the skin dose averaging area, particularly in relation to hot particle exposure, was considered. We considered the issue in practical application of regulation and concept of dosimetry with the help of dose distribution data derived from electron point kernel. Also, some options for rationalization of the issue were discussed.

\section{Issue on Dose Averaging Area}

The ICRP Main Commission set a skin dose limit of 0.5 Gy over an area of $1 \mathrm{~cm}^{2}$ at $70 \mu \mathrm{m}$ in ICRP Publication $\left.60 .{ }^{6}\right)$ From mid 1980s, exposure from hot particles became an issue among nuclear industries and regulatory bodies particularly in the United States. ${ }^{7}$ In mid 1990s, both the United States Nuclear Regulatory Commission (USNRC) and nuclear industries conducted research projects to improve knowledge on the effects of hot particles on skin. Concerns were expressed on high potential of violation against the skin dose limit in case of hot particle contamination on skin. ${ }^{8}$ In 1999, NCRP published Report 130 after an intensive review of available intensive review of available scientific information, ${ }^{1)}$ where NCRP recommended $10 \mathrm{~cm}^{2}$ for the dose averaging area instead of $1 \mathrm{~cm}^{2}$ to mitigate this concern. The Position Statement 9 of NCRP $^{9)}$ followed in 1991 supporting the recommendations in NCRP 130. This position was adopted in 10CFR20 Standards for protection against radiation in 2002. ${ }^{10)}$

In spite of the position change in the United States, the position of ICRP remains unchanged. In its new recommendations, ICRP keeps the same position as that of ICRP Publication 60, that the skin dose should be assessed at $70 \mu \mathrm{m}$ of depth over the most highly exposed $1 \mathrm{~cm}^{2}{ }^{21)}$ Incidentally, size of the dose averaging area is of no concern for relatively uniform area contamination. However, since skin 
dose from a hot particle is concentrated in small area, much smaller than $1 \mathrm{~cm}^{2}$, averaging over $10 \mathrm{~cm}^{2}$ reduces the skin dose to $1 / 10$ of dose averaged over $1 \mathrm{~cm}^{2}$. This is evident in Table 1 which shows skin doses from a point source on skin calculated for several radionuclides averaged over the two different areas at the same depth of $70 \mu \mathrm{m}$ using a computer code, K-SKIN. ${ }^{12)}$

Table. 1 Comparison of beta dose rate calculated with K-SKIN averaged over $1 \mathrm{~cm}^{2}$ and $10 \mathrm{~cm}^{2}$ at $70 \mu \mathrm{m}$ from point source of $1 \mathrm{~Bq}$

\begin{tabular}{|c|c|c|c|c|}
\hline \multirow{2}{*}{ Nuclides } & \multirow{2}{*}{$\begin{array}{l}\mathrm{E}_{\beta, \max } \\
(\mathrm{MeV})\end{array}$} & \multicolumn{2}{|c|}{ Dose averaging area } & \multirow{2}{*}{$\begin{array}{c}\text { Ratio } \\
\left(10 \mathrm{~cm}^{2} / 1 \mathrm{~cm}^{2}\right)\end{array}$} \\
\hline & & $1 \mathrm{~cm}^{2}$ & $10 \mathrm{~cm}^{2}$ & \\
\hline Pm-147 & 0.23 & $5.28 \mathrm{E}-4$ & $5.28 \mathrm{E}-5$ & 0.10 \\
\hline Tc-99 & 0.29 & $1.07 \mathrm{E}-3$ & $1.07 \mathrm{E}-4$ & 0.10 \\
\hline Cs-137 & 0.51 & $1.31 \mathrm{E}-3$ & $1.31 \mathrm{E}-4$ & 0.10 \\
\hline Sr-90 & 0.55 & $1.26 \mathrm{E}-3$ & $1.26 \mathrm{E}-4$ & 0.10 \\
\hline $\mathrm{Cl}-36$ & 0.71 & $1.35 \mathrm{E}-3$ & $1.35 \mathrm{E}-4$ & 0.10 \\
\hline Tl-204 & 0.76 & $1.15 \mathrm{E}-3$ & $1.15 \mathrm{E}-4$ & 0.10 \\
\hline Y-90 & 2.28 & $1.39 \mathrm{E}-3$ & $1.25 \mathrm{E}-4$ & 0.09 \\
\hline $\mathrm{Mn}-56$ & 2.85 & $1.28 \mathrm{E}-3$ & $1.08 \mathrm{E}-4$ & 0.08 \\
\hline
\end{tabular}

\section{Distribution of Doses from a Hot Particle}

Skin dose distribution across the radius of dose averaging area was constructed. To calculate dose distribution, beta energy spectrum of various nuclides ${ }^{13)}$ and electron point kernels calculated from Monte Carlo techniques were employed. ${ }^{14)}$ Fig.1 shows the dose profiles for different nuclides emitting beta particles of different maximum energies. Including relatively high energy beta particles (e.g.

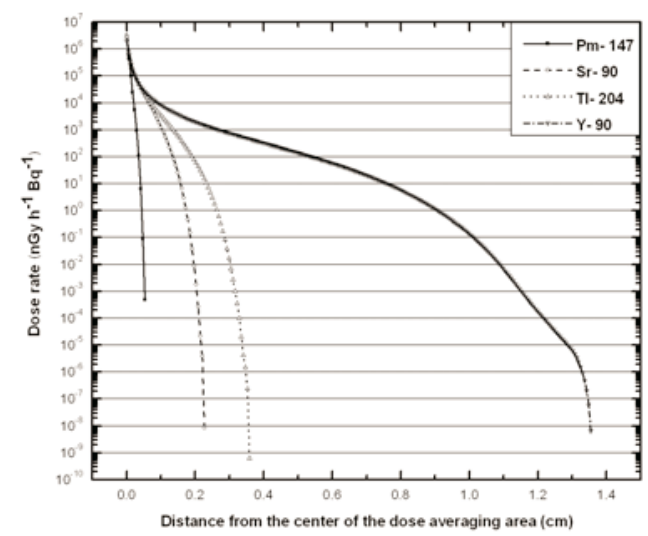

Fig. 1 Distribution of skin absorbed dose across the radius of dose averaging area. Over $99 \%$ of the dose incurred with in $1 \mathrm{~mm}$ radius.

Y-90), over $99 \%$ of transferred energies from nuclides were finished within $1 \mathrm{~mm}$.

Therefore, averaging over $10 \mathrm{~cm}^{2}$ (radius of $1.78 \mathrm{~cm}$ ) simply makes the resulting skin dose one tenth of that averaged over $1 \mathrm{~cm}^{2}$. The NCRP explained as the basis of widening the dose averaging area that over $90 \%$ of hot particle contamination is found on clothing rather than on the skin so that actual area of skin exposed to the hot particle is much wider due to changes of relative positions of the hot particle against the central area and variation of distance from the hot particle to the skin surface. ${ }^{1)}$ However, this explanation is no more than a excuse for the deliberate over-averaging to meet the regulatory dose limit of skin.

\section{Assessment of Options for Controlling Localized Skin Dose}

The skin dose limit, $0.5 \mathrm{~Sv}$ averaged over $1 \mathrm{~cm}^{2}$, is translated as 1010 beta particles from a particle on a skin, the limiting time for a $3 \mathrm{MBq}$ hot particle is around 55 minutes. ${ }^{15}$ ) This means that workers in hot particle prone area should be monitored every hour because possibility of contamination by such an intense hot particle is there although it would be rare. Such a close monitoring of workers incurs significant cost and psychological burden to the workers. This implies that the regulatory practice, based on skin dose limit recommended by ICRP, put severe burden to the licensees in monitoring hot particles and should be avoided.

One option for controlling localized skin dose is the one taken by the USNRC; increasing the dose averaging area. Application of $10 \mathrm{~cm}^{2}$ instead of $1 \mathrm{~cm}^{2}$ practically reduces dose by factor 10, as shown above, in case of small area contamination like a hot particle. Hence for a small area contamination on skin, averaging over $10 \mathrm{~cm}^{2}$ provides relaxation of the dose limit by 10 times, i.e. up to $5 \mathrm{~Gy}$ in case of contaminated area less than $1 \mathrm{~cm}^{2}$. In other words, extension of the dose averaging area results the same effects as an increase in dose limit although the numerical value $(0.5$ Gy or $500 \mathrm{mSv}$ ) is unchanged.

The USNRC's approach to control skin exposure which is based on the recommendations of the NCRP would be a practical resolution of the concerns on hot particle exposures. However it may compromise the concept of dosimetry by intentionally including unexposed volume of tissue in dose calculation to reduce the average dose. In certain macrodosimetric conditions, an averaging over a volume including non-targeted cells or region of an organ is accepted mainly for the purpose of controlling stochastic risk. Examples are dose assessments for widely distributed tissues like red bone marrow or for internal emitter very localized in an organ. The main concern related to the skin is the deterministic effects rather than cancer induction. For the purpose of protection against deterministic effects, particularly in localized exposure, the local dose is more appropriate quantity, if it can be reasonably assessed, than the dose averaged over larger volume.

There could be another option that gives some flexibility in implementation of the dose equivalent limit on skin particularly for highly localized skin exposure. This means adoption of a graded enforcement of the skin dose limit in national regulations. For examples, a localized exposure above the dose limit $(0.5 \mathrm{~Sv})$ but not exceeding $5 \mathrm{~Sv}$ could 
be classified as a "reportable incident" while an exposure beyond $5 \mathrm{~Sv}$ comprises a violation against the regulations.

This option is founded on two assumptions. The first assumption is that hot particle exposures are rare enough to be regarded as an incident. Kelly and Gustafson surveyed all the operating nuclear power plants in the United States and got reports on over 15000 hot particles. ${ }^{16)}$ Among the reported particles, only $0.2 \%$ (around 30) involved contamination directly on skin with activities higher than 40 $\mathrm{kBq}$. The second assumption is that a very localized skin exposure below 5 Gy dose not result important health effects According to the NCRP's evaluation, when small areas of skin are irradiated by hot particle sufficiently to cause erythema and lesions accompanying dry desquamation, such effects are temporary, are confined to an area of a few square millimeters and are not considered to be severe deterministic effects. ${ }^{1)}$ Ulceration, dermal thinning and pigment changes in such small areas at deeper depth are also not considered to be severe deterministic effects. The ICRP also agreed with this view by recommending $1 \mathrm{~Sv}$ over an area of $1 \mathrm{~cm}^{2}$ to prevent acute transient ulceration and $5 \mathrm{~Sv}$ to prevent the acute deep ulceration as the dose restriction in ICRP 59, ${ }^{3)}$ although the final recommendations given in ICRP $60,{ }^{6}$ ) simply set the dose equivalent limit to skin as $0.5 \mathrm{~Sv}$ averaged over any $1 \mathrm{~cm}^{2}$ with no further explanations.

Hence, the above option applying relaxation of skin dose limit for hot particle exposure may compromise the dose limitation system recommended by the ICRP. Nevertheless, it can be an alternative to the option averaging doses over wider area which compromises the concept of dosimetry.

Another option is revisiting the dose limit if there is a room for setting higher dose limits. The dose limit to skin, recommended by ICRP, is for protection of the skin against harmful deterministic effects. Even if the assessment of NCRP that a skin dose up to 5 Gy is tolerable is accepted, the limit on skin dose should be much less to meet the effective dose limits. Since the share of skin in the effective dose should remain in a small portion, e.g. $30 \%$ which is equivalent to $6 \mathrm{mSv}$ a year, then the skin dose equivalent is limited $600 \mathrm{mSv}$ because the tissue weighting factor of skin is 0.01 . This implies that the equivalent dose limit to skin cannot be increased much higher than the current limit recommended by ICRP without some changes in the dose limitation concept for skin.

Under the current concept, assuming workers exposed to hot particles also subject to whole body exposure via externally or internally, allowance of skin dose should be limited to around $0.5 \mathrm{~Sv}$ to meet the effective dose limit since the skin dose is evaluated for the most highly exposed $1 \mathrm{~cm}^{2}$ regardless of the exposed area. The reason why the ICRP reduced the dose averaging area is to prevent the deterministic effects, not the stochastic effects, in small areas receiving highly localized doses. Since the effective dose limits are for controlling the stochastic risk, we may use different dose averaging areas for the two purposes; a small area (e.g. $1 \mathrm{~cm}^{2}$ as it is) for effective dose assessment and a larger area (e.g. $10 \mathrm{~cm}^{2}$ or even $100 \mathrm{~cm}^{2}$ ) for equivalent dose assessment. With the $0.5 \mathrm{~Sv}$ of skin dose limit, ICRP stated that the guidance given in ICRP Publcation $35^{17)}$ on averaging area for monitoring of skin contamination, 100 $\mathrm{cm}^{2}$, is still valid. Then the equivalent dose limit for prevention of deterministic effects can be increased to certain higher level, $5 \mathrm{~Sv}$ for instance.

The first two options, one lowering dose by increasing the dose averaging area while maintaining the dose limit and the other applying graded enforcement while keeping the averaging area, are choices of a national regulatory body. The third option belongs to jurisdiction of the ICRP.

\section{Conclusion}

The issue of skin dose averaging area was critically reviewed with the help of dose distribution data calculated using electron point kernel and K-SKIN code, particularly in relation to hot particle exposure. It appeared that both a small area and a larger area, e.g. $10 \mathrm{~cm}^{2}$ proposed by NCRP, over which skin doses are averaged have pros and cons in concept of dosimetry and in sense of practical applications.

To draw and propose a position on the dose averaging area for which a discrepancy remains in the recommendations between the ICRP and the NCRP, two other options were suggested and discussed. One option addresses flexibility in enforcement of the skin dose limit but compromises the unified meaning of the dose limits. The other option proposed two different dose limits for controlling deterministic effects and stochastic effects, respectively. Namely, increase of the skin equivalent dose limit specifically for prevention of deterministic effects while applying a smaller averaging area for prevention of stochastic effects.

From the review on the dose averaging area issue where potentials of hot particles to cause exposure exceeding the skin dose limits is high, it was concluded that a rationale on this important issue is very needed.

\section{Acknowledgment}

This work was supported by the Korea Hydro \& Nuclear Power Company, Ltd.

\section{References}

1) M. W. Charles and J. D. Harrison, "Hot particle dosimetry and radiobiology-past and present," J. Radiol. Prot., 27, 97 (2007).

2) B. J. Dionne and J. W. Baum, "Discrete radioactive particles at nuclear power plants: Prevention, mitigation and control," Radiat. Prot. Manag., 8, 65 (1991).

3) International commission on Radiological Protection, The Biological Basis for Skin Dose Limitation, ICRP Publication 59, Ann. ICRP 22(2), Pergamon Press, Oxford, (1991).

4) National Council on Radiation Protection and Measurements, Biological Effects and Exposure Limits for "Hot Particles", NCRP Report No.130, (NCRP), (1999).

5) M. W. Charles, "The Skin in Radiological Protection-Recent Advances and Residual Unresolved Issues," Radiat. Prot. Dosim., 109[4], 323 (2004).

6) International commission on Radiological Protection, 1990 Recommendations of the International Commission on 
Radiological Protection, ICRP Publication 60, Ann. ICRP 21(1-3), Pergamon Press, Oxford, (1991).

7) United States Nuclear Regulatory Commission, Proposed Rules, 66 FR 134, USNRC, (2001)

8) United States Nuclear Regulatory Commission, Information Notice 90-48, Enforcement policy for hot particle exposure, 55 FR 31113, USNRC, (1990).

9) National Council on Radiation Protection and Measurements, Extension of the skin dose limit for hot particles to other external sources of skin irradiation, NCRP Statement No.9, (NCRP), (2001).

10) United States Nuclear Regulatory Commission, Final Rules, 67 FR 66, USNRC, (2002).

11) International Commission on Radiological Protection, The 2007 Recommendations of the International Commission on Radiological Protection, ICRP Publication 103, Pergamon Press Oxford, (2007).
12) S. H. Park, B. Choi and J. L. Lee, "Development of point-kernel code for skin dose calculation," Nuclear Technol., 168, 158 (2009).

13) International Commission on Radiological Protection, Radionuclides Transformations: Energy and Intensity of Emissions, ICRP Publication 38, Pergamon Press, Oxford, (1981).

14) S. M. Seltzer, "Electron-Photon Monte Carlo calculations: The ETRAN code,” J. Appl. Radiat. Isot., 42, 9 (1991).

15) United States Nuclear Regulatory Commission, Regulatory analysis of revision to 10 CFR 20: Unified Skin Dose Limit, USNRC, (2001).

16) J. J. Kelly and S. Gustafson, Industry Experience with Discrete Radioactive Particles, EPRI Report TR-104125, Electric Power Research Institute (EPRI), (1994).

17) International Commission on Radiological Protection, General Principles of Monitoring for Radiation Protection of Workers, ICRP Publication 35, Pergamon Press, Oxford, (1982). 